\title{
Clinical Characteristics and Outcome in Elderly Patients with Traumatic Brain Injury: For Establishment of Management Strategy
}

\author{
Hiroshi KARIBE, ${ }^{1}$ Toshiaki HAYASHI, ${ }^{1}$ Ayumi NARISAWA, ${ }^{1}$ \\ Motonobu KameYAma, ${ }^{1}$ Atsuhiro NAKAGAWA, ${ }^{2}$ and Teiji TOMINAGA ${ }^{2}$ \\ ${ }^{1}$ Department of Neurosurgery, Sendai City Hospital, Sendai, Miyagi, Japan; \\ ${ }^{2}$ Department of Neurosurgery, Tohoku University Graduate School of Medicine, \\ Sendai, Miyagi, Japan
}

\begin{abstract}
In recent years, instances of neurotrauma in the elderly have been increasing. This article addresses the clinical characteristics, management strategy, and outcome in elderly patients with traumatic brain injury (TBI). Falls to the ground either from standing or from heights are the most common causes of TBI in the elderly, since both motor and physiological functions are degraded in the elderly. Subdural, contusional and intracerebral hematomas are more common in the elderly than the young as the acute traumatic intracranial lesion. High frequency of those lesions has been proposed to be associated with increased volume of the subdural space resulting from the atrophy of the brain in the elderly. The delayed aggravation of intracranial hematomas has been also explained by such anatomical and physiological changes present in the elderly. Delayed hyperemia/hyperperfusion may also be a characteristic of the elderly TBI, although its mechanisms are not fully understood. In addition, widely used pre-injury anticoagulant and antiplatelet therapies may be associated with delayed aggravation, making the management difficult for elderly TBI. It is an urgent issue to establish preventions and treatments for elderly TBI, since its outcome has been remained poor for more than 40 years.
\end{abstract}

Key words: traumatic brain injury (TBI), elderly, delayed deterioration, antiplatelet, anticoagulant

\section{Introduction}

According to the Annual Report on the Aging Society 2015 from the cabinet office of the Japan government, ${ }^{1)}$ the aged (65 y.o. or older) population in Japan is approximately $33,920,000$ in 2015 , as its percentage against whole population is $26.7 \%$. It is predicted that the aged population will rise to reach its peak as $39,000,000$ in 2040. The rate of aged population will rise afterwards to reach to its peak as $40 \%$ in 2060 .

As aged population increase, instances of traumatic brain injury (TBI) in the elderly are increasing. It has been known that the frequency curve of TBI by age groups has two peaks, as both 15-29 and 65-79 y.o. ${ }^{2)}$ In recent years, it is going to have a single peak only at the elderly generation, resulting from both decreased frequency in the young and increased frequency in the elderly. ${ }^{3)}$ In addition, the peak of frequency in the elderly is going to shift to older. ${ }^{4)}$

Received March 2, 2017; Accepted April 18, 2017
Such changes may be resulted from population aging, as well as recent reduction in traffic injury.

In general, prognostic factors of TBI includes prior history of medical illness, multiple traumatic injuries, severity of injury, mechanism of injury, clinical severity such as Glasgow Coma Scale (GCS) score, pupillary response, hypoxia, hypotension, and so on. Age has been proposed as one of the most reliable prognostic factors following TBI. ${ }^{5}$ Both survival and functional outcome are significantly poorer in the elderly compared to the younger patients with TBI. It has been also reported that the duration of hospital stay is significantly longer in the elderly than younger patients with TBI. ${ }^{6}$ According to Japan Neurotrauma Data Bank (JNTDB), it has been reported that the mortality seems to decrease in the elderly with TBI, without increase of favorable functional outcome. ${ }^{4)}$ The increase of elderly patients with TBI may require the increase of medical, rehabilitation, and nursing care resources. Thus, it is an urgent and an important issue to establish preventions and treatments of elderly TBI for Japan as well as other 
developing countries, which are facing both falling birthrates and aging societies.

In this article, both the clinical characteristics and the problems of TBI in the elderly, including delayed deterioration or the influences of either anti-coagulant or anti-platelet, are reviewed to make reference to the management strategy and the outcome.

\section{Clinical Characteristics and Problems of TBI in the Elderly}

\section{Characteristics and problems of physical function in the elderly}

As the cause of TBI, falls to the ground either from standing or from heights are the most common causes of TBI in the elderly, whereas the traffic accident is less common. ${ }^{7)}$ The cause of falls may be closely associated with the deterioration of physical functions, including visual, auditory, and equilibrium functions. The weakened muscle strength or the articular diseases may be also included, as well as cognitive dysfunction by dementia, faint, cardiac hypofunction, orthostatic hypotension, hemiparesis resulting from stroke, anemia, hypoglycemia, dehydration, body temperature elevation due to systemic infections. In addition, certain drugs have been identified as "fall risk-increasing drugs", such as opioids, antipsychotics, anxiolytics, hypnotics, sedatives, and antidepressants. Drugs, which may cause or worsen orthostatism, may also modify to increase the risk of fall, such as vasodilators used in cardiac diseases, antihypertensives, diuretics, beta blocking agents, calcium channel blockers, reninangiotensin system inhibitors, alpha-adrenoreceptor antagonists, dopaminergic agents, and so on. ${ }^{8)}$ In certain situations, it is important that treatments for either such causes of trauma or backgraound diseases may be given priority for the management of TBI in the elderly.

Pedestrian accidents are significantly more common in the elderly than the young, as the second common next to falls. They account for $13 \%$ of the TBI mechanisms in the elderly, being high risk for severe TBI. ${ }^{9)}$ Impaired cognitive function, such as either concentration or reaction, may be associated with the mechanisms of injury, as well as deterioration of physical and physiological functions.

\footnotetext{
Morphological characteristics and pathophysiology

Morphologically, distribution of traumatic intracranial lesion is different by age groups. Diffuse injury is less common in the elderly than the young. In contrast, focal injury is more common in the elderly. ${ }^{10-12)}$ It is well known that the subdural,
}

contusional, and intracerebral hematomas are more common TBI lesions in the elderly than the young, although epidural hematomas are less common in the elderly. Those characteristics of intracranial lesion have been explained by morphological changes due to aging. It has been reported that whole brain volume increases during childhood, and reaches to its peak during young adulthood between 18 and 35 years of age. Whole brain volume then decreases gradually after 35 years of age, as $0.2-0.5 \%$ per year. Approximately, $6-11 \%$ of brain volume reduction occurs by $60-80$ years of age. ${ }^{13)}$

As the brain volume decreases in the elderly, the volume of subdural space increases as well as the mobility of the cerebral hemisphere. In addition to the fragility of the vascular wall structure by aging, since bridging veins adhere to the dura mater or to the brain surface firmly, bridging veins may be at risk to be injured by cerebral hemisphere mobilization by trauma, resulting in subdural hematomas. Increase of cerebral hemispheric mobility may also cause contre-coup injury. The fragility of cerebral vessels by aging may cause intracerebral hematomas in the elderly. In contrast, epidural hematomas rarely occur in the elderly, since the dura mater adheres inner surface of the cranium.

Interhemispheric subdural hematoma (iSDH) is a rare but a typical clinical entity with which the increased volume of the subdural space is closely associated (Fig. 1). It typically occurs in the elderly by the injury of bridging veins which flow into the superior sagittal or the straight sinuses. The amount of the iSDH is usually small to be expected spontaneous healing in most cases. Some cases with a large amount of iSDH often represents falx syndrome, which typically show contralateral hemiparesis or monoparesis of lower extremity. The iSDH is commonly appear at parietal and occipital region, and rarely complicated with skull fractures. ${ }^{14,15)}$

\section{Physiological characteristics and the influences of systemic complication}

From the view point of cerebral blood flow (CBF) and metabolism, CBF at cerebral cortex decreases by aging, although subcortical CBF is not significantly

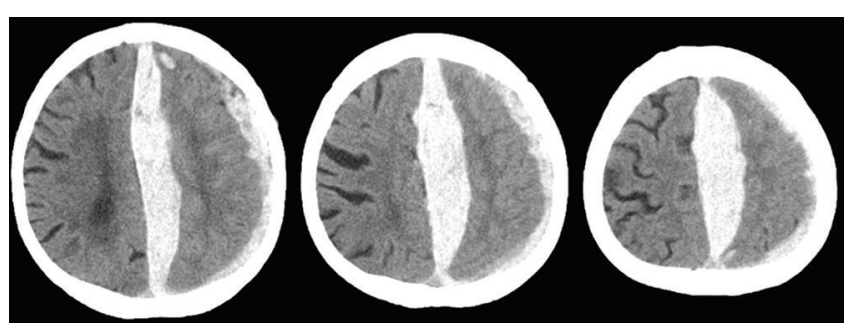

Fig. 1 Interhemispheric subdural hematoma (iSDH). 
affected by age. ${ }^{16,17)}$ Cerebral arterial autoregulation is impaired in the elderly, as well as vasoreactivity to either $\mathrm{pCO}_{2}$ or metabolic changes. ${ }^{18,19)}$ In addition, since the tolerance to ischemia is impaired by aging, the elderly has been postulated to be vulnerable to reduced CBF and metabolism after TBI.

CBF reduction during the first several hours after $\mathrm{TBI}^{20,21)}$ may be brought by cerebral perfusion pressure (CPP) decrease due to the elevation of intracranial pressure (ICP), cerebral vasospasm, and dysautoregulation of cerebral arteries. Thus, either ICP or CPP oriented management has been thought to be important for TBI, to avoid secondary ischemic injury. Since autoreguation of cerebral vessels are impaired in elderly patients with TBI in general, CPP management may be more suitable to be oriented.

Hyperemia/hyperperfusion may occur subsequent to several hours period of cerebral hypoperfusion. ${ }^{20-22)}$ Since hyperemia/hyperperfusion is often prolonged and closely associated with poor outcome, reperfusion injury may play some roles in delayed deterioration. ${ }^{23)}$ Early induction of mild hypothermia has been reported to be useful for the management of traumatic intracranial hematoma. ${ }^{24)}$ Thus, mild hypothermia may have a potential as a treatment option for reperfusion injury induced by hyperemia/hyperperfusion in TBI. However, it has been reported that hypothermia failed to improve outcome in patients over 45 years of age with TBI, ${ }^{25}$ suggesting that an optimal treatment strategy has not been established yet.

From the aspect of systemic physiological function, subclinical cardio-pulmonary dysfunction by aging reduces systemic physiological reserve capacity as well as tolerance to traumatic stress, leading to secondary traumatic injury. For example, subclinical pulmonary dysfunction easily induces hypoxia in the elderly with TBI. ${ }^{26,27)}$ Subclinical cardiac dysfunction limits safety range of treatment between hypovolemia and volume overload. ${ }^{28)}$ In addition, pathophysiological conditions after TBI may be harmfully influenced by certain systemic complications such as malignant tumors, renal dysfunction, liver diseases, cardiac diseases, chronic obstructive pulmonary diseases, and so on. ${ }^{29,30)}$ These systemic physiological dysfunction, even subclinical, may harmfully influence on the outcome in the elderly cases with not only severe but also mild to moderate TBI.

\section{Delayed deterioration in the elderly}

Survival curve after TBI by age groups seems interesting, ${ }^{6}$ as the elderly patients show a markedly different trend in comparison with other age groups. Death within 48 hours of admission is not different among all age groups. After then, only the elderly group shows a progressive decrease in survival until the rate leveled off at approximately $35 \%$, whereas the other age groups tend to level off at approximately $60-80 \%$ survival. ${ }^{6}$ These results suggest that delayed deterioration may be an important cause of poor outcome in the elderly with TBI.

Delayed deterioration, so-called "talk and deteriorate (T\&D)", is defined as a patient who represent a relatively good neurological condition during the first certain period after head injury, and then deteriorate to a severe neurological condition (e.g. Glasgow Coma Scale score of 8 or less) within 48 hours of injury. It is more common in the elderly than young, and has been proposed as occurring in $20-30 \%$ of the elderly patients with TBI. T\&D is often induced by delayed hyperemia/hyperperfusion, delayed traumatic intracerebral hematoma, delayed expansion of subdural hematoma, or delayed aggravation of traumatic contusional edema. The enlarged subdural space, which buffers ICP elevation, may delay to represent clinical deterioration in the elderly. In addition, cognitive disorders such as dementia can also delay the detection of neurological deterioration in the elderly.

Delayed deterioration is the most common in elderly cases with cerebral contusion. ${ }^{31,32)}$ Cerebral contusional edema has been classified as early massive edema and delayed-pericontusional edema. ${ }^{33)}$ The early massive edema is defined as a hypointensity core on diffusion-weighted magnetic resonance image (MRI), which appears within 24 hours after TBI, suggesting that intra- and extracellular components undergo disintegration and homogenization in the central area of the contusion. In contrast, delayedpericontusional edema is defined as predominant cellular swelling in the peripheral area. There is a crescent-shaped border zone of very high acquisition diffusion coefficient value on MRI between these central and peripheral area. ${ }^{33,34)}$ In spite of CBF reduction, edema fluid is supplied excessively in the area of the cerebral contusion. In addition, hyperemia/hyperperfusion subsequent to ischemia enhances contusional edema aggravation, resulting in delayed deterioration.

Delayed traumatic intracerebral hematoma (Fig. 2) and delayed expansion of either traumatic acute subdural hematoma (Fig. 3) or intracerebral hematoma (Fig. 4) may be other important clinical entities for delayed deterioration. Either of them may delay to represent clinical neurological deterioration, since the expansion of hematomas can be masked by the buffering effect of enlarged subdural space. In addition, the intracranial tissue compliance is declined in the elderly. ${ }^{35)}$ Thus, the delayed deterioration 

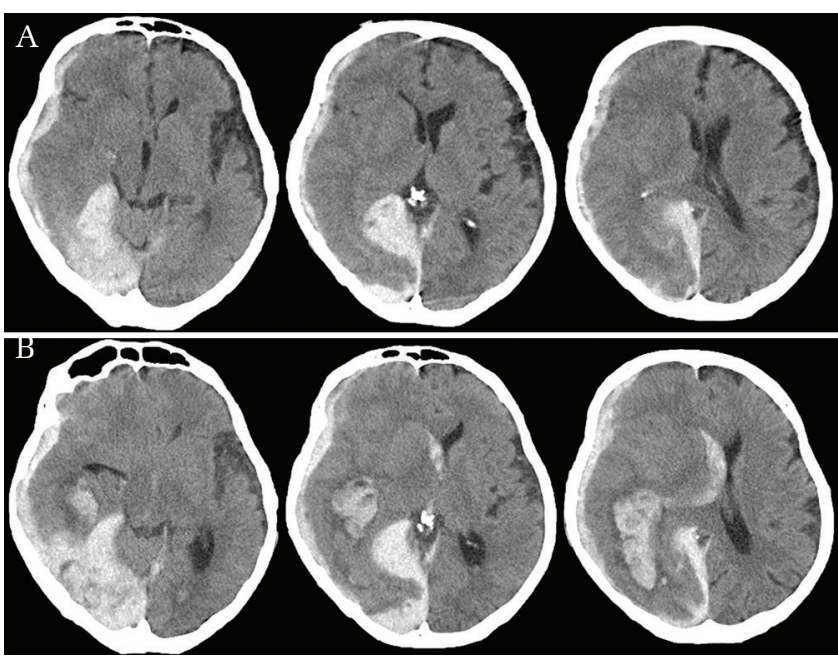

Fig. 2 Delayed traumatic intracerebral hematoma. Note: (A) A falco-tentorial and convexity acute subdural hematoma (ASDH) was evident on CT at 1 hour after trauma. (B) The ASDH thickened slightly at 6 hours after trauma. An additional subcortical intracerebral hematoma appeared at right posterior-temporal area.
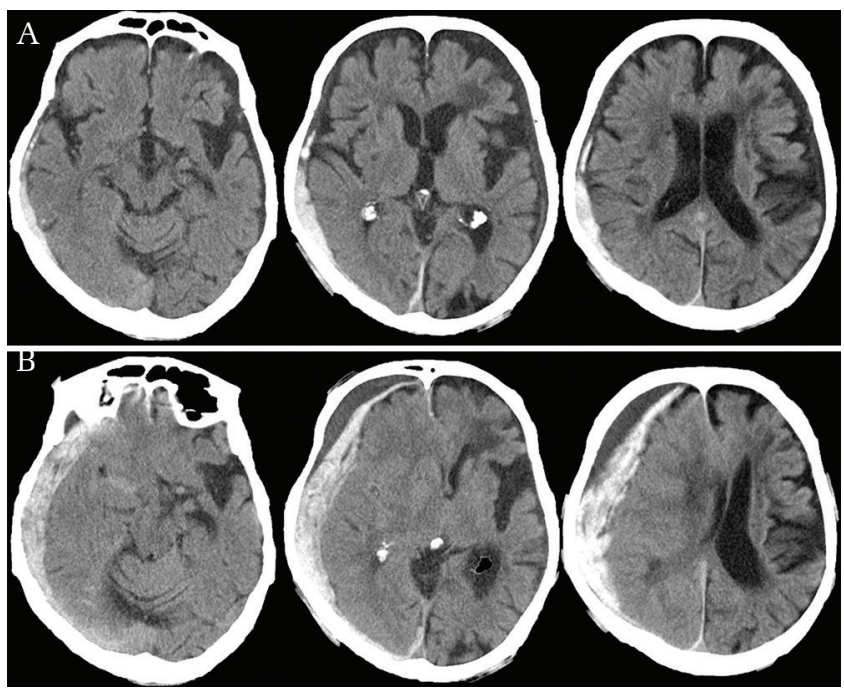

Fig. 3 Delayed expansion of ASDH. Note: (A) A thin ASDH was evident on CT at 1 hour after trauma. (B) The hematoma thickened at 6 hours after trauma.

is rapidly progressive when enlarged hematomas exceed the intracranial buffering capacity.

As a unique entity of delayed deterioration after TBI, "delayed posttraumatic acute subdural hematoma (DASH)" in elderly patients on anticoagulation has been reported. ${ }^{36)}$ DASH has been defined as an acute subdural hematoma that is not apparent on the initial computed tomography (CT), and appears on a follow-up CT after 9 to 72 hours after TBI. ${ }^{36,37)}$ Thus, DASH should be suspected in elderly, anticoagulated, mild TBI patients including those who
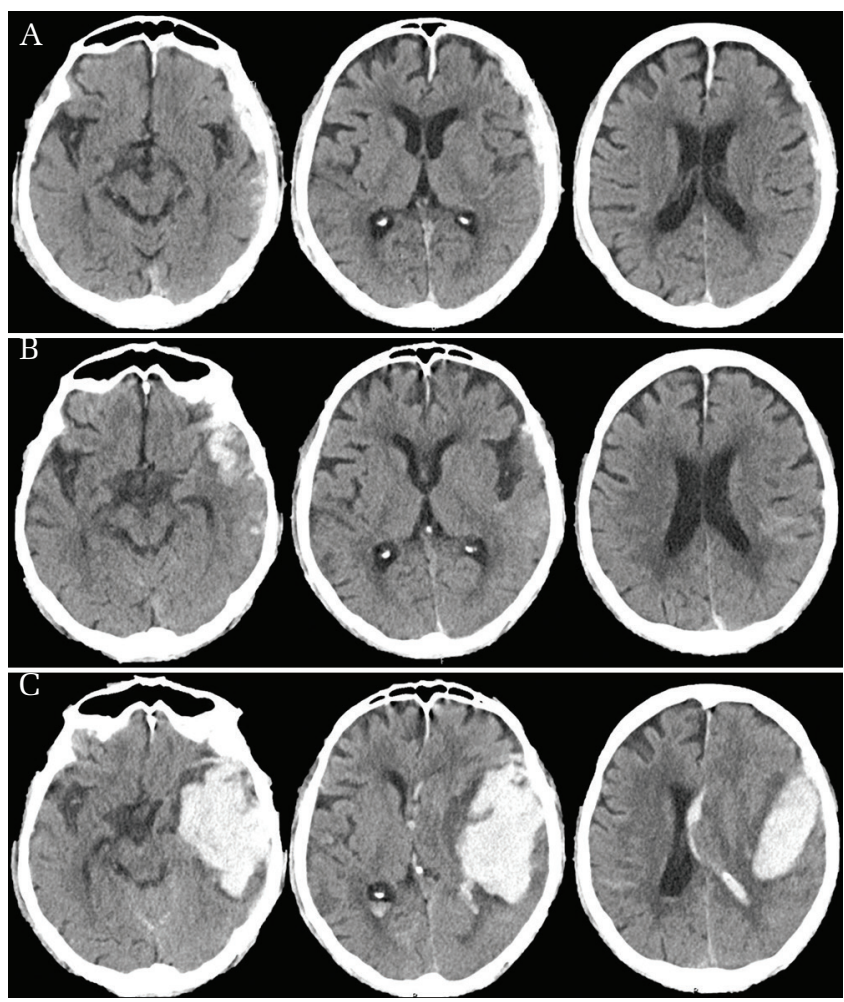

Fig. 4 Delayed expansion of traumatic intracerebral hematoma. Note: (A) Only a thin ASDH was evident on CT at 1 hour after trauma. (B) A small contusional hematoma appeared at $\mathbf{2 4}$ hours after trauma, although the initial ASDH was mostly washed out. (C) The contusional hematoma was fused and enlarged at 48 hours after trauma.

present with Glasgow Coma Scale Scores of 15, and normal initial CT. It has been suggested that elderly anticoagulated mild TBI patients should be admitted for 24 to 48 hours of observation after injury.

\section{Influences of anticoagulants and antiplatelets}

Anticoagulants, such as warfarin, has been widely used to prevent thrombo-embolisms in patients with atrial fibrillation, prosthetic cardiac valve replacement, or deep venous thrombosis, and so on. In recent years, warfarin has been replaced by novel direct anticoagulants (DOACs) in a certain extent of cases, since DOACs do not require routine monitoring and reduce hemorrhagic complication compared with warfarin. Antiplatelets, such as aspirin, cilostazol, and clopidogrel, have been widely used to prevent thrombo-embolisms in patients with ischemic heart disease, ischemic stroke, arteriosclerosis obliterans, and so on. Patients, who have a history of thromboembolic disease mentioned above, are basically at a risk of either falls or traffic accidents. ${ }^{38,39)}$ Although there are a few reports that neither anticoagulant 
nor antiplatelet influence on the mortality and morbidity in patients with $\mathrm{TBI}^{40-42)}$ most of the available data suggest that a pre-injury reception of either anticoagulant or antiplatelet agent increases the risk of poor outcome in patients with TBI, mainly resulting from larger or repeated intracranial hematoma. ${ }^{4-52)}$ It has been also reported that the history of anticoagulant is closely associated with the occurrence of talk and deteriorate. ${ }^{53)}$

Therefore, in patients who receive pre-injury antiplatelet or anticoagulant therapy, discontinuation and/or reversal of these drugs should be considered after TBI, especially in cases with traumatic intracranial hematomas. Table 1 summarizes available anti-platelets and anti-coagulant and their reversal agents (Table 1). Careful consideration is necessary to determine discontinuation and/or reversal of these drugs, since the risk for thrombo-embolism is different in each patient or background diseases.

For example, in cases with coronary stent implantation, the risk of life-threatening stent thrombosis is increased 30 -fold if clopidogrel is discontinued within the first 30 days. ${ }^{54)}$ Discontinuation of clopidogrel within the first 6-months is an independent determinant of stent thrombosis. ${ }^{55)}$ It has been also reported that the cardiac complication rate is threefold increased after aspirin withdrawal. ${ }^{56)}$ In patients who require surgery, it has been demonstrated that the risk of coronary thrombosis after discontinuation of antiplatelet is greater than the risk of surgical bleeding if antiplatelet is continued.57) "Bridging therapy" with heparin has been reported as ineffective in reducing cardiac events after withdrawal of antiplatelet therapy in patients with cardiac stents. ${ }^{58)}$

As another example, preinjury use of warfarin seems to increase mortality in elderly patients regardless of injury severity ${ }^{59)}$ The admitted prothrombin timeinternational normalized ratio (PT-INR) is directly correlated with mortality. ${ }^{49)}$ Specific replacement therapy, including vitamin $\mathrm{K}$, fresh frozen plasma, or VII factor, facilitates successful clot evacuation without bleeding complications. ${ }^{60)}$ Discontinuation of warfarin has been reported as approximately $5-6 \%$ per year risk of thromboembolism in cases with non-valver atrial fibrillation. ${ }^{61)}$

Regarding DOACs, it has been reported that they are associated with lower mortality in patients with traumatic intracranial hemorrhage. ${ }^{62)}$ However, reversal agents have not been established in most of DOACs except dabigatran (Table 1). In addition, both benefits and disadvantages have not been well known regarding discontinuation and/or reversal of DOACs in elderly cases with TBI.

Therefore, whether to discontinue anti-coagulant/platelet therapy or not in patients with TBI may be decisions that depend on a variety of circumstances, such as the evidence mentioned above, the extent of the traumatic intracranial hematoma, and the indication for pre-injury use of anti-coagulant/-platelet agents. Currently, both risks and benefits, which are brought by discontinuation and/or reversal of either one of the anticoagulant or antiplatelet, should be carefully considered in each case. ${ }^{63)}$

\section{Establishment of management strategy}

As described above, the characteristics of the elderly with TBI suggest certain ideal strategies for the management. For example, the elderly with TBI may be easily affected by hypoxia or hypotension, since cardio-pulmonary reserve capacity is limited in the elderly. Shortening of either rescue or transfer time may be required to minimize harmful effect of hypoxia or hypotension, prehospitally. Careful assessment and/or monitoring of systemic condition may be also required for adequate cardio-pulmonary management, such as physiological monitoring

Table 1 Anti-platelet/-coagulant and potential reversal agents

\begin{tabular}{|c|c|c|c|}
\hline Medication & Mechanism of action & Duration of effect & Potential reversal agents \\
\hline Aspirin & Irreversible COX-1 inhibition & $\begin{array}{l}>36 \text { hrs until } 20 \% \text { baseline after } \\
\text { discontinuation }\end{array}$ & $\begin{array}{l}\text { Platelets, desmopressin, } \\
\text { rFVIIa }\end{array}$ \\
\hline Clopidogrel, Ticlopidine & $\begin{array}{l}\text { Platelet ADP receptor (P2Y12) } \\
\text { antagonist }\end{array}$ & $\begin{array}{l}5 \text { days to baseline after } \\
\text { discontinuation }\end{array}$ & $\begin{array}{l}\text { Platelets, desmopressin, } \\
\text { rFVIIa }\end{array}$ \\
\hline Cilostazol & PDE-III inhibitor & $\begin{array}{l}48 \text { hrs to baseline after } \\
\text { discontinuation }\end{array}$ & Platelets, desmopressin \\
\hline Warfarin & $\begin{array}{l}\text { Inhibit vitamin } \mathrm{K} \text { dependent } \\
\text { clotting factors (II, VII, IX, X) }\end{array}$ & $\begin{array}{l}48-72 \text { hrs to baseline after } \\
\text { discontinuation }\end{array}$ & Vitamin K, FFP, rFVIIa \\
\hline Dabigatran & Thrombin inhibitor & & Idarucizumab \\
\hline Rivaroxaban & Factor Xa inhibitor & $24 \mathrm{hr}$ & - \\
\hline Apixaban & Factor Xa inhibitor & & - \\
\hline Edoxaban & Factor Xa inhibitor & $24 \mathrm{hr}$ & - \\
\hline
\end{tabular}


including electrocaridogram, blood pressure, pulseoxymetry, repeated blood-gas analyses, and so on. Since delayed deterioration seems closely correlated with outcome, early detection or prediction of delayed intracranial changes may be also important. To achieve it, monitoring of ICP, CPP, or other intracranial physiological variables may be important, as well as repeated careful neurological inspection and CT examination. In general, since the elderly may be risky for both general anesthesia and craniotomy, a less invasive procedure such as endoscopic acute subdural hematoma (ASDH) evacuation may be an ideal tool to minimize surgical complication. ${ }^{64)}$

In Guidelines for the Management of Severe Head Injury 3rd Edition, edited by the Japan Society of Neurotraumatology in 2013, the following recommendations are stated as experts' opinion regarding elderly with TBI; (1) prehospital care, initial treatment, and ICU management are equivalent to those for general adults, (2) strict and repeated neurological inspection is desirable to detect "talk and deteriorate", (3) systemic complications should be optimally treated, (4) both early induction and extended period of rehabilitation are desirable, since functional recovery after TBI often takes longer in the elderly, (5) surgical indications for the elderly are equivalent to those for general adults, although they should be carefully applied. ${ }^{65)}$ Such evidencebased guidelines for TBI have not stated any specific recommendation to the elderly, regarding prehospital care, primary management, or either one of timing, indications, or techniques of surgical management, despite age has been identified as a reliable independent prognostic factor for TBI. Prospective trial will be necessary to clarify evidences regarding those in future.

\section{Conflicts of Interest Disclosure}

None of the authors have any conflicts of interest (COI) associated with this study. All authors who are members of The Japan Neurosurgical Society (JNS) have registered online Self-reported COI Disclosure Statement Forms through the website for JNS members.

\section{References}

1) Cabinet office: Annual report on the aging society 2015. http://www8.cao.go.jp/kourei/english/ annualreport/2015/2015pdf_e.html (Accessed on 2017 March 1)

2) Kameyama M, Karibe H, Onuma $T$, Tominaga $T$ : Epidemiological study of head injury in Miyagi Neurotrauma Data Bank: age, cause of injury, pathophysiology and outcome. Neurotraumatology 31: 49-56, 2008 (Japanese)

3) Kameyama M, Karibe H, Kawase M, Hayashi T, Hirano T, Tominaga T: Severe head injury and age in Japan Neurotrauma Data Bank: comparison among project 1998, 2004, and 2009. Neurotraumatology 36: 10-16, 2013 (Japanese)

4) Yokohori S, Araki T, Onda H, et al.: Transition of aggressive treatment and patient outcome in geriatric severe traumatic brain injury: an analysis from the Japan Neurotrauma Data Bank (JNTDB) project 1999, 2004, and 2009. Neurotraumatology 36: 76-85, 2013 (Japanese)

5) Howard MA, Gross AS, Dacey RG, Winn HR: Acute subdural hematomas: an age-dependent clinical entity. J Neurosurg 71: 858-863, 1989

6) Jane JA, Francel PC: Age and outcome of head injury. In Narayan RK, Wilberger JE Jr, Povlishock JT (eds): Neurotrauma. New York, McGraw-Hill, 1996, pp. 793-804.

7) Kannus P, Palvanen M, Niemi S, et al.: Increasing number and incidence of fall-induced severe head injuries in older adults: nationwide statistics in Finland in 1970-1995 and prediction for the future. Am J Epidemiol 149: 143-150, 1999

8) Milos V, Bondesson $\AA$, Magnusson M, Jakobsson U, Westerlund T, Midlöv P: Fall risk-increasing drugs and falls: a cross-sectional study among elderly patients in primary care. BMC Geriatr 14: 40,2014

9) Champion HR, Copes WS, Buyer D, Flanagan ME, Bain L, Sacco WJ: Major trauma in geriatric patients. Am J Public Health 79: 1278-1282, 1989

10) Eisenberg HM, Gary HE, Aldrich EF, et al.: Initial CT findings in 753 patients with severe head injury. A report from the NIH Traumatic Coma Data Bank. J Neurosurg 73: 688-698, 1990

11) Ono J, Isobe $K$, Watanabe $Y$, Yamaura A: Clinical problems in the management of aged patients with severe head injury: analysis of neurological findings and CT findings. No Shinkei Geka 21: 717-721, 1993 (Japanese)

12) Tokutomi $T$, Ogawa $T$, Ono J, et al.: Intracranial diagnosis according to the Traumatic Coma Data Bank classification of computed tomography imaging in the Japan Neurotrauma Data Bank. Neurotraumatology 28: 1-5, 2005 (Japanese)

13) Hedman AM, van Haren NE, Schnack HG, Kahn RS, Hulshoff Pol HE: Human brain changes across the life span: a review of 56 longitudinal magnetic resonance imaging studies. Hum Brain Mapp 33: 1987-2002, 2012

14) Urculo E, Martinez L, Gereka L, Olasagasti V, Olascoaga J, Urcola J: The spontaneous reabsorbtion of posttraumatic interhemispheric subdural haematoma. Acta Neurochir (Wien) 138: 776-777, 1996

15) Takeuchi S, Takasato $Y$, Masaoka H, Hayakawa T, Yatsushige H, Sugawara T: Traumatic interhemispheric subdural haematoma: Study of 35 cases. J Clin Neurosci 17: 1527-1529, 2010 
16) Meyer JS, Terayama Y, Takashima S: Cerebral circulation in the elderly. Cerebrovasc Brain Metab Rev 5: 122-146, 1993

17) Chen JJ, Rosas HD, Salat DH: Age-associated reductions in cerebral blood flow are independent from regional atrophy. Neuroimage 55: 468-478, 2011

18) Czosnyka M, Balestreri $M$, Steiner L, et al.: Age, intracranial pressure, autoregulation, and outcome after brain trauma. J Neurosurg 102: 450-454, 2005

19) Peisker T, Bartoš A, Skoda O, Ibrahim I, Kalvach P: Impact of aging on cerebral vasoregulation and parenchymal integrity. J Neurol Sci 299: 112-115, 2010

20) Bouma GJ, Muizelaar JP, Choi SC, Newlon PG, Young HF: Cerebral circulation and metabolism after severe traumatic brain injury: the elusive role of ischemia. J Neurosurg 75: 685-693, 1991

21) Marion DW, Darby J, Yonas H: Acute regional cerebral blood flow changes caused by severe head injuries. J Neurosurg 74: 407-414, 1991

22) Karibe H, Kameyama M, Nimura T, et al.: Symptomatic hemispheric hyperperfusion in elderly patients with traumatic head injury. Progress in Computed Imaging 31: 255-262, 2009 (Japanese)

23) Chieregato A, Noto A, Tanfani A, Bini G, Martino C, Fainardi E: Hyperemia beneath evacuated acute subdural hematoma is frequent and prolonged in patients with an unfavorable outcome: a xe-computed tomographic study. Neurosurgery 64: 705-717; discussion 717-718, 2009

24) Clifton GL, Coffey CS, Fourwinds S, et al.: Early induction of hypothermia for evacuated intracranial hematomas: a post hoc analysis of two clinical trials. J Neurosurg 117: 714-720, 2012

25) Clifton GL, Miller ER, Choi SC, et al.: Lack of effect of induction of hypothermia after acute brain injury. $N$ Engl J Med 344: 556-563, 2001

26) Pudelek B: Geriatric trauma: special needs for a special population. AACN Clin Issues 13: 61-72, 2002

27) Deiner S, Silverstein JH, Abrams KJ: Management of trauma in the geriatric patient. Curr Opin Anaesthesiol 17: 165-170, 2004

28) Demetriades D, Karaiskakis M, Velmahos G, et al.: Effect on outcome of early intensive management of geriatric trauma patients. Br J Surg 89: 1319-1322, 2002

29) Grossman MD, Miller D, Scaff DW, Arcona S: When is an elder old? Effect of preexisting conditions on mortality in geriatric trauma. J Trauma 52: 242-246, 2002

30) Yilmaz S, Karcioglu O, Sener S: The impact of associated diseases on the etiology, course and mortality in geriatric trauma patients. Eur J Emerg Med 13: 295-298, 2006

31) Lobato RD, Rivas JJ, Gomez PA, et al.: Head-injured patients who talk and deteriorate into coma. Analysis of 211 cases studied with computerized tomography. J Neurosurg 75: 256-261, 1991

32) Kawamata $T$, Katayama Y: Head injured patients who talk and deteriorate: analysis of 86 cases registered on the Japan Neurotrauma Databank. Neurotraumatology 25: 205-09, 2002 (Japanese)

33) Katayama Y, Kawamata T: Mechanisms of early formation in cerebral contusion. Neurotraumatology 23: 6-9, 2000

34) Ito J, Marmarou A, Barzó P, Fatouros P, Corwin F: Characterization of edema by diffusion-weighted imaging in experimental traumatic brain injury. J Neurosurg 84: 97-103, 1996

35) Albeck MJ, Skak C, Nielsen PR, Olsen KS, Børgesen SE, Gjerris F: Age dependency of resistance to cerebrospinal fluid outflow. J Neurosurg 89: 275-278, 1998

36) Itshayek E, Rosenthal G, Fraifeld S, Perez-Sanchez X, Cohen JE, Spektor S: Delayed posttraumatic acute subdural hematoma in elderly patients on anticoagulation. Neurosurgery 58: E851-856; discussion E851-E856, 2006

37) Cohen T, Gudeman S: Delayed traumatic intracranial hematomas. Neurotrauma, Narayan RK, Wilberger JE Jr., Povlishock JT (eds): New York, McGraw-Hill, 1996, pp. 689-702

38) Ferrera PC, Bartfield JM: Outcomes of anticoagulated trauma patients. Am J Emerg Med 17: 154-156, 1999

39) Gaetani P, Revay M, Sciacca S, et al.: Traumatic brain injury in the elderly: considerations in a series of 103 patients older than 70. J Neurosurg Sci 56: 231-237, 2012

40) Rozzelle CJ, Wofford JL, Branch CL: Predictors of hospital mortality in older patients with subdural hematoma. J Am Geriatr Soc 43: 240-244, 1995

41) Wojcik R, Cipolle MD, Seislove E, Wasser TE, Pasquale MD: Preinjury warfarin does not impact outcome in trauma patients. J Trauma 51: 1147-1151; discussion 1151-1152, 2001

42) Bonville DJ, Ata A, Jahraus CB, et al.: Impact of preinjury warfarin and antiplatelet agents on outcomes of trauma patients. Surgery 150: 861-868, 2011

43) Karni A, Holtzman R, Bass T, et al.: Traumatic head injury in the anticoagulated elderly patient: a lethal combination. Am Surg 67: 1098-1100, 2001

44) Mina AA, Knipfer JF, Park DY, Bair HA, Howells GA, Bendick PJ: Intracranial complications of preinjury anticoagulation in trauma patients with head injury. J Trauma 53: 668-672, 2002

45) Lavoie A, Ratte S, Clas D, et al.: Preinjury warfarin use among elderly patients with closed head injuries in a trauma center. J Trauma 56: 802-807, 2004

46) Ohm C, Mina A, Howells G, Bair H, Bendick P: Effects of antiplatelet agents on outcomes for elderly patients with traumatic intracranial hemorrhage. J Trauma 58: 518-522, 2005

47) Cohen DB, Rinker C, Wilberger JE: Traumatic brain injury in anticoagulated patients. J Trauma 60: 553-557, 2006

48) Franko J, Kish KJ, O’Connell BG, Subramanian S, Yuschak JV: Advanced age and preinjury warfarin anticoagulation increase the risk of mortality after head trauma. J Trauma 61: 107-110, 2006 
49) Pieracci FM, Eachempati SR, Shou J, Hydo LJ, Barie PS: Degree of anticoagulation, but not warfarin use itself, predicts adverse outcomes after traumatic brain injury in elderly trauma patients. J Trauma 63: 525-530, 2007

50) Ivascu FA, Howells GA, Junn FS, Bair HA, Bendick PJ, Janczyk RJ: Predictors of mortality in trauma patients with intracranial hemorrhage on preinjury aspirin or clopidogrel. J Trauma 65: 785-788, 2008

51) Wong DK, Lurie F, Wong LL: The effects of clopidogrel on elderly traumatic brain injured patients. J Trauma 65: 1303-1308, 2008

52) Inamasu J, Nakatsukasa M, Kuramae T, Nakagawa $Y$, Miyatake S, Tomiyasu K: Influence of age and antiplatelet/anti-coagulant use on the outcome of elderly patients with fall-related traumatic intracranial hemorrhage. Neurol Med Chir (Tokyo) 50: 1051-1055, 2010

53) Davis DP, Kene M, Vilke GM, et al.: Head-injured patients who "talk and die": the San Diego perspective. J Trauma 62: 277-281, 2007

54) Moussa ID, Colombo A: Antiplatelet therapy discontinuation following drug-eluting stent placement: dangers, reasons, and management recommendations. Catheter Caridovasc Interv 74: 1047-1054, 2009

55) Airoldi F, Colombo A, Morici N, et al.: Incidence and predictors of drug-eluting stent thrombosis during and after discontinuation of thienopyridine treatment. Circulation 116: 745-754, 2007

56) Biondi-Zoccai GG, Lotrionte M, Agostoni P, et al.: A systematic review and meta-analysis on the hazards of discontinuing or not adhering to aspirin among 50,279 patients at risk for coronary artery disease. Eur Heart J 27: 2667-2674, 2006

57) Di Minno MN, Prisco D, Ruocco AL, Mastronardi P, Massa S, Di Minno G: Perioperative handling of patients on antiplatelet therapy with need for surgery. Intern Emerg Med 4: 279-288, 2009

58) Vicenzi MN, Meislitzer T, Heitzinger B, Halaj M, Fleisher LA, Metzler H: Coronary artery stenting and non-cardiac surgery-a prospective outcome study. Br J Anaesth 96: 686-693, 2006

59) McMillian WD, Rogers FB: Management of prehospital antiplatelet and anticoagulant therapy in traumatic head injury: a review. J Trauma 66: 942-950, 2009

60) Senft C, Schuster T, Forster MT, Seifert V, Gerlach $\mathrm{R}$ : Management and outcome of patients with acute traumatic subdural hematomas and preinjury oral anticoagulation therapy. Neurol Res 31: 1012-1018, 2009

61) Patel MR, Hellkamp AS, Lokhnygina Y, et al.: Outcomes of discontinuing rivaroxaban compared with warfarin in patients with nonvalvular atrial fibrillation: analysis from the ROCKET AF trial (Rivaroxaban Once-Daily, Oral, Direct Factor Xa Inhibition Compared With Vitamin K Antagonism for Prevention of Stroke and Embolism Trial in Atrial Fibrillation). J Am Coll Cardiol 61: 651-658, 2013

62) Feeney JM, Santone E, DiFiori M, Kis L, Jayaraman V, Montgomery SC: Compared to warfarin, direct oral anticoagulants are associated with lower mortality in patients with blunt traumatic intracranial hemorrhage: A TQIP study. J Trauma Acute Care Surg 81: 843-848, 2016

63) Beynon C, Hertle DN, Unterberg AW, Sakowitz OW: Clinical review: Traumatic brain injury in patients receiving antiplatelet medication. Crit Care 16: 228, 2012

64) Yokosuka K, Uno M, Matsumura K, et al.: Endoscopic hematoma evacuation for acute and subacute subdural hematoma in elderly patients. J Neurosurg 123: 1065-1069, 2015

65) The Guideline Committee on the Management of Severe Head Injury: Severe traumatic head injury in the elderly. Guidelines for the Management of Severe Head Injury 3rd Edition, Tokyo, Igakushoin, 2013, pp. 150-154 (Japanese)

Address reprint requests to: Hiroshi Karibe, MD, Department of Neurosurgery, Sendai City Hospital, 1-1-1 Asuto-nagamachi, Taihaku-ku, Sendai, Miyagi 982-8502, Japan.

e-mail: karibe@nsg.med.tohoku.ac.jp 